\title{
Cesare Cantù, Condizione enonomica delle lettere
}

\section{Marco Stupazzoni}

\section{(2) OpenEdition \\ 1 Journals}

\section{Edizione digitale}

URL: https://journals.openedition.org/studifrancesi/7282

DOI: $10.4000 /$ studifrancesi.7282

ISSN: 2421-5856

\section{Editore}

Rosenberg \& Sellier

\section{Edizione cartacea}

Data di pubblicazione: 1 avril 2010

Paginazione: 167

ISSN: 0039-2944

\section{Notizia bibliografica digitale}

Marco Stupazzoni, «Cesare Cantù, Condizione enonomica delle lettere», Studi Francesi [Online], 160 (LIV | I) | 2010, online dal 30 novembre 2015, consultato il 20 novembre 2021. URL: http://

journals.openedition.org/studifrancesi/7282 ; DOI: https://doi.org/10.4000/studifrancesi.7282

Questo documento è stato generato automaticamente il 20 novembre 2021.

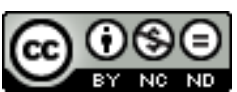

Studi Francesi è distribuita con Licenza Creative Commons Attribuzione - Non commerciale - Non opere derivate 4.0 Internazionale. 


\title{
Cesare Cantù, Condizione enonomica delle lettere
}

\author{
Marco Stupazzoni
}

\section{NOTIZIA}

CESARE CANTÙ, Condizione enonomica delle lettere, a cura di Marinella COLUMNI CAMERINO, in Cesare Cantù e dintorni, a cura di Matilde DILLON WANKE e Luca BANI, Milano, CisalpinoIstituto Editoriale Universitario, 2007, «Biblioteca dell'archivio storico lombardo», pp. 125-168.

1 Apparso in tre puntate come articolo nella «Rivista Europea» tra il 25 aprile e il 15 maggio 1838, questo lucido e severo studio di Cesare Cantù sulla condizione economica delle lettere in Europa, ma soprattutto in Italia, nella prima metà dell'Ottocento, è riproposto all'attenzione degli studiosi all'interno della miscellanea di studi dedicati all'illustre storico e scrittore lombardo presentati in occasione del Convegno bergamasco del 27 aprile 2006.

2 Nella prima parte del suo saggio, Cantù conduce una meticolosa indagine sulla situazione della produzione libraria e del mercato editoriale nei vari paesi (europei ed extraeuropei) e focalizza la sua attenzione sugli aspetti legislativi e giudirici che, in misura differente e spesso sproporzionata, garantiscono o negano, nei diversi stati, il diritto di proprietà letteraria. Occupandosi più dettagliatamente delle «garanzie concesse dalla legge di Francia alla proprietà letteraria mettendo gli scrittori in agiata situazione» (p. 139), l'autore cita numerosi esemi di autori che egli ritiene favoriti da tali circostanze: Chateaubriand, Lamartine, Hugo, Vigny, Soulié, Janin, Sand (la quale «tocca dalla "Revue des deux mondes" fino 1000 franchi ogni articolo lungo o breve») e Balzac che «pare invece scaduto alquanto di prezzo» (p. 137). E, ancora a proposito dell'autore della Comédie humaine, Cantù osserva che Balzac, «imbriacando continuamente il pubblico, guadagnò tesori, sebbene altrettanti ne spendesse, a segno che spesso fu costretto a rimettere i suoi creditori al nuovo romanzo» (p.139). 
Tutt'altro che positivo è il quadro tracciato dall'autore in relazione alla situazione italiana: «la condizione economica dei letterati italiani, scrive Cantù, è ben lontana dall'offrire un prospero aspetto» (p. 141). Innumerevoli sono, a suo giudizio, le ragioni di questo stato di arretratezza e di inefficienze, prima fra tutte la suddivisione dell'Italia in stati troppo disomogenei e squilibrati tra loro nelle leggi riguardanti la proprietà letteraria. Tale frammentazione politico-legislativa, che impedisce altresì «il rapido circolare dei libri nel paese istesso» (p. 145), si manifesta anche nella «mancanza di accordo» (p. 158), di unione e di coerenza tra i diversi ambienti letterati italiani. Per queste ragioni, Cantù auspica che, per far rifiorire la vita letteraria in Italia, sia assolutamente necessaria la «cooperazione degli studiosi e del pubblico, di chi fa i libri, di chi li compra, di chi li legge, e di chi giudica senza leggerli» (p. 163). 